\title{
By Targeting Stat3 microRNA-17-5p Promotes Cardiomyocyte Apoptosis in Response to Ischemia Followed by Reperfusion
}

\author{
Weijie Du Zhenwei Pan Xu Chen Leimin Wang Ying Zhang Shuang Li \\ Haihai Liang Chaoqian Xu Yong Zhang Yanping Wu Hongli Shan Yanjie Lu \\ Department of Pharmacology (Key Laboratory of Cardiovascular Medicine Research, Ministry of \\ Education; State-Province Key Laboratories of Biomedicine-Pharmaceutics of China), Harbin Medical \\ University, Harbin, Heilongjiang, People's Republic of China
}

\section{Key Words}

Apoptosis • Ischemia reperfusion injury $\bullet \operatorname{miR}-17-5 p \cdot$ Stat3

\begin{abstract}
Background: Several studies have confirmed the role of microRNAs in regulating ischemia/ reperfusion-induced cardiac injury (I/R-I). MiR-17-5p has been regarded as an oncomiR in the development of cancer. However, its potential role in cardiomyocytes has not been exploited. The aim of this study is to investigate the role of miR-17-5p in I/R-I and the underlying mechanism through targeting Stat3, a key surviving factor in cardiomyocytes. Methods: MTT (3-[4, 5-dimethylthiazol-2-yl]-2, 5 diphenyl tetrazolium bromide) assay was used to detect the cell viability. ELISA and TUNEL were performed to measure apoptosis of neonatal rat ventricular cardiomyocytes (NRVCs). Infarct area was estimated by TTC (triphenyltetrazolium chloride) and Evans blue staining. Western blot analysis was employed to detect the Stat3 and p-Stat3 levels and real-time RT-PCR was used to quantify miR-17-5p level. Results: The miR17-5p level was significantly up-regulated in I/R-I mice and in NRVCs under oxidative stress. Overexpression of miR-17-5p aggravated cardiomyocyte injury with reduced cell viability and enhanced apoptotic cell death induced by $\mathrm{H}_{2} \mathrm{O}_{2}$, whereas inhibition of miR-17-5p by its antisense AMO-17-5p abrogated the deleterious changes. Moreover, the locked nucleic acidmodified antisense (LNA-anti-miR-17-5p) markedly decreased the infarct area and apoptosis induced by I/R-I in mice. Furthermore, overexpression of miR-17-5p diminished the $p$-Stat3 level in response to $\mathrm{H}_{2} \mathrm{O}_{2}$. The results from Western blot analysis and luciferase reporter gene assay confirmed Stat3 as a target gene for miR-17-5p. Conclusion: Upregulation of miR-17$5 p$ promotes apoptosis induced by oxidative stress via targeting Stat 3 , accounting partially for I/R-I.
\end{abstract}

Copyright $(2014$ S. Karger AG, Basel 
Du et al.: miRNA-17-5p Promotes Cardiomyocyte Apoptosis

\section{Introduction}

Myocardial infarction is a leading cause of death worldwide, accompanied by apoptosis, cardiac fibrosis, inflammation and pathological remodeling [1]. Reperfusion is the definitive treatment for acute coronary syndromes, especially acute myocardial infarction; however, reperfusion has the potential to exacerbate tissue injury, a process termed myocardial ischemia/reperfusion injury (I/R-I) [2]. Excessive production of reactive oxygen species (ROS) to create oxidative stress is the key damaging factor to the heart during I/R-I, as reoxygenation during reperfusion generates a large amount of ROS as an apoptosis inducer [3-5]. Though multiple signaling pathways and molecular players are involved in cardiac I/R-I, the precise molecular mechanisms remain incompletely understood.

There is mounting evidence that apoptosis plays a critical role in the evolution of I/R-I and contributes to cardiomyocyte loss in the border zone and in remote myocardium in the early phase, as well as months after myocardial infarction, leading to pathological remodeling and heart failure [6, 7].

MicroRNAs (miRNAs), a newly discovered class of small non-protein-coding mRNAs, have rapidly emerged as the key player of the regulatory network of gene expression. Through post-transcriptional regulation, these molecules fine-tune the gene expression to define the proteome of a cell to produce diverse cellular functions and well-defined biological phenotypes $[8,9]$. Several miRNAs have been implicated in the regulation of pathological and physiological process of heart disease [10-12].

MiR-17-5p is a member of miR-17-92 cluster, located on the human chromosome 13q31, which is a polycistronic miRNA gene encoding six miRNAs $[13,14]$. Expression profiling studies have revealed widespread overexpression of miR-17-5p in diverse tumor subtypes including both hematopoietic malignancies and solid tumors such as those derived from breast, colon and lung $[15,16]$. However, based on the previous studies it appears that miR17-5p may have distinct effects in different kinds of tumors. MiR-17-5p exhibited oncogenic activity via downregulating retinoblastoma 1 , a well established tumor suppressor in thyroid cancer cells [17]. Conversely, in breast cancer, tumor-suppressive effect of miR-17-5p was attributed to directly targeting oncogenes [18]. Moreover, the hearts of miR-17-5p 92deficient mutant embryos presented a clear ventricular septal defect, indicating a critical role of miR-17-5p 92 miRNAs in cardiac development [19]. Li et al. found that miR-17-5p was activated during kidney ischemia-reperfusion injury in mice [20]. Signal transducer and activator of transcription-3 (Stat3), a key cellular survival factor, plays an important antiapoptotic role in response to oxidative stress $[21,22]$. In addition, the $3^{\prime}$ UTR of the Stat 3 gene containing the predicated target sites for miR-17-5p was identified using several miRNA databases. There is a possibility therefore that miR-17-5p might contribute to shaping the I/R-I via modulating apoptotic cell death through targeting Stat3. However, such a possibility has not been tested.

In this study, we first investigated changes of miR-17-5p expression in a mouse model of I/R-I and a cellular model of oxidative stress induced by $\mathrm{H}_{2} \mathrm{O}_{2}$. We then assessed the effects of miR-17-5p on apoptosis in both in vivo and in vitro models. We finally established Stat 3 as a cognate target gene for miR-17-5p. We also revealed that inhibition of miR-17-5p by its specific antisense inhibitor preserved cell survival and rescued cell death in both I/R-I and cellular oxidative stress models.

\section{Materials and Methods}

\section{Animals}

Adult male C57BL/ 6 mice used in this study were kept under controlled conditions (humidity: 55 $\pm 5 \%$; temperature: $23 \pm 1{ }^{\circ} \mathrm{C}$ and a 12 -h light/dark artificial cycle). All experimental procedures were in accordance to the Institutional Animal Care and Use Committee of Harbin Medical University, P.R. China. The protocol was approved by the Experimental Animal Ethic Committee of Harbin Medical University. 
Mouse model of myocardial ischemia/reperfusion injury

Thirty healthy adult male C57BL/6 mice were randomly divided into sham-operated and age-matched control, I/R-I, and I/R-I+LNA-17-5p groups. The animals were anesthetized by intraperitoneal (i.p.) injection of pentobarbital ( $60 \mathrm{mg} / \mathrm{kg}$ ). The heart was exposed by a left-sided thoracotomy. I/R-I model was established by ligating the left anterior descending artery (LAD) $2-3 \mathrm{~mm}$ from the tip of the left auricle using a 7/0 polypropylene suture tied with a plastic cannula for $30 \mathrm{~min}$ followed by reopening of the artery for $24 \mathrm{~h}$. The sham animals underwent an identical procedure with a suture placed around LAD but without LAD ligation. The occlusion and reperfusion were confirmed by regional cyanosis of the myocardial surface and ST-segment elevation in lead II of the ECG monitored with subcutaneous stainless steel electrodes. After I/R-I procedure, the air was expelled from the chest and the surgical wounds were sutured closed. To prevent infection, penicillin was applied systemically. Finally, the animals were placed on a heating pad within the cage until full recovery of consciousness [23, 24]. At the end of the myocardial I/R protocols, about $0.5 \mathrm{ml}$ of $5 \%$ Evans blue dye (Sigma-Aldrich St Louis, USA) was injected into the vena cava to delineate the region of myocardial perfusion. After remaining blood had been washed out and the right ventricle been trimmed out, the left ventricle was cut into 2 -mm-thick slices and stained with $1 \%$ triphenyltetrazolium chloride (TTC) for $10 \mathrm{~min}$ at $37^{\circ} \mathrm{C}$. The infarct area was identified by non-staining region while the live area should turn red. The area at risk (AAR; ischemic area) and infarct area were calculated using the Image ProPlus 5.0 software. The ischemic tissues were collected and stored at $-80^{\circ} \mathrm{C}$ for later uses.

\section{Synthesis and administration of locked nucleic acid-modified antisense}

The antisense sequence of miR-17-5p (LNA-antimiR-17-5p) was synthesized by Exiqon (Denmark) and five nucleotides or deoxynucleotides at both ends of the antisense molecules were locked (LNA; the ribose ring is constrained by a methylene bridge between the 2-0-and the 4-Catoms). The sequence of LNAantimiR-17-5p is 5'-GCACTGTAAGCACTTT-3'. LNA-antimiR-17-5p was intravenously injected via tail vein into mice at a dose of $1 \mathrm{mg} / \mathrm{kg}$ two days before cardiac I/R.

\section{Primary culture of neonatal rat ventricular myocytes}

Primary neonatal rat ventricular cardiomyocyte (NRVCs) from 1 to 3-day-old SD rats were prepared by trypsin as previously described. Briefly, hearts were removed aseptically from the rats that had been decapitated. Ventricle tissues were cut into small pieces and trypsinized at $37^{\circ} \mathrm{C}$ for $10 \mathrm{~min}$, and this procedure was repeated for 4 times. The cell suspension was centrifuged at $1000 \mathrm{rpm}$ for $5 \mathrm{~min}$ and resuspended in medium for $2 \mathrm{~h}$. Then the non-adherent cardiomyocytes were removed and plated in 24-well plates with DMEM medium (Invitrogen, Carlsbad, USA) containing 10\% fetal bovine serum (Hyclone, Logan, USA), 2 $\mathrm{mM}$ L-glutamate, $100 \mathrm{U} / \mathrm{ml}$ penicillin $\mathrm{G}, 100 \mu \mathrm{g} / \mathrm{ml}$ streptomycin, and $0.1 \mathrm{mM}$ bromodeoxyuridine (SigmaAldrich St Louis, USA) at $37^{\circ} \mathrm{C}$ in $5 \% \mathrm{CO}_{2}$ and $95 \%$ air.

Transfection procedure and $\mathrm{H}_{2} \mathrm{O}_{2}$ treatment

After starvation in serum-free medium for $12 \mathrm{~h}$, the miRNA and Lipofectamine 2000 (Invitrogen, Carlsbad, USA) were separately mixed with Opti-MEM ${ }^{\circledR}$ I Reduced Serum Medium (Gibco, New York, USA) for $5 \mathrm{~min}$. Then, the two mixtures were combined and incubated for $20 \mathrm{~min}$ at room temperature. Next, the mixtures were added to the cells and incubated at $37^{\circ} \mathrm{C}$ for $12 \mathrm{~h}$. Finally, $2 \mathrm{ml}$ medium containing $10 \%$ FBS was added to the 6-well plates and maintained under the normal cultured condition. $\mathrm{H}_{2} \mathrm{O}_{2}(100 \mu \mathrm{M})$ was added to cells and incubated for $12 \mathrm{~h}$.

\section{Luciferase reporter gene activity assay}

The $3^{\prime}$ UTR of Stat3 gene containing the predicated target sites for miR-17-5p was obtained by PCR amplification. The fragment was inserted into the multiple cloning sites in the pMIR-REPORT luciferase miRNA expression reporter vector (Ambion, Austin, USA). HEK-293 cells were co-transfected with $0.1 \mu \mathrm{g}$ of luciferase reporters containing Stat3 3'UTR and miR-17-5p mimics by Lipofectamine 2000 (Invitrogen, Carlsbad, USA). We harvested the cell lysates after $48 \mathrm{~h}$ transfection and measured the luciferase activity with a dual luciferase reporter assay kit according to manufacturer's instruction. 
Du et al.: miRNA-17-5p Promotes Cardiomyocyte Apoptosis

\section{Real-time RT-PCR}

Total RNA samples from heart tissues and cultured cells were extracted using Trizol reagent (Invitrogen, Carlsbad, USA). RNA $(0.5 \mu \mathrm{g})$ was then reverse-transcribed using high-capacity cDNA reverse transcription kit (Applied Biosystems, Foster City, USA) to obtain first-strand cDNA. MiR-17-5p level was quantified by the mirVana qRT-PCR miRNA detection kit (Ambion, Austin, USA) in conjunction with realtime PCR with SYBR Green as described previously [25]. After circle reaction, the threshold cycle (Ct) was determined and relative miR-17-5p level was calculated based on the Ct values and normalized to U6 level in each sample [26].

\section{MTT Assay}

MTT assay was used to evaluate survival of cells with miR-17-5p or $\mathrm{H}_{2} \mathrm{O}_{2}$ treatment. The cells were plated at 10,000 cells per $\mathrm{cm}^{2}$ in 96-well plates. After treatment, $20 \mu \mathrm{l}$ of MTT (Sigma-Aldrich St Louis, USA) was added to each well, and incubated with the cells for $4 \mathrm{~h}$ at $37^{\circ} \mathrm{C}$. The supernatant was removed and the cell layer was dissolved in $150 \mu$ DMSO. The plate was shaken for $15 \mathrm{~min}$ for solubilization of crystals and the optical density of each well was determined using a model 680 microplate reader at $490 \mathrm{~nm}$ (Bio-Rad, Hercules, USA) [27].

\section{Apoptosis analysis}

Apoptosis of cardiac myocytes was detected by staining mouse heart cryosections or culture cells with the TUNEL fluorescence FITC kit (Roche, Indianapolis, USA). Quantification of DNA fragment (by measuring optical density) was performed by enzyme-linked immunosorbent assay (ELISA) on the basis of antibody detection of free histone and fragmented DNA. After TUNEL staining, the heart sections or cells were immersed into DAPI (Sigma-Aldrich, St Louis, USA) solution to stain nuclei. Fluorescence staining was viewed by a laser scanning confocal microscope (FV300, Olympus, Tokyo, Japan).

\section{Western blot analysis}

Total protein sample was extracted from the tissues or the cultured cells with the procedures as described in detail [25]. Briefly, protein samples $(80 \mu \mathrm{g})$ were separated in SDS-PAGE and blotted to nitrocellulose membrane. The membrane was incubated with primary antibodies including Stat3 (Cell Signaling Technology, Boston, USA), p-Stat3 (phospho-Stat3 at Tyr 705; Cell Signaling Technology, Boston, USA) and $\beta$-actin (Kangcheng Shanghai, China), and then secondary antibodies (Alexa Fluor; Molecular Probes, Eugene USA). The bands were quantified using Odyssey v1.2 software (Gene Company Limited, Hong Kong, China) by measuring the band intensity (area×OD) for each group and normalizing to $\beta$-actin as an internal control.

\section{Statistical analysis}

All data are presented as mean \pm SEM. One-way ANOVA followed by Bonferroni or Dunnett's post hoc test was used for multiple comparisons and Student's $t$-test for two-group comparisons as appropriate. A two-tailed $p<0.05$ was considered statistically significant. Data were analyzed using the GraphPad Prism 5.0 software.

\section{Results}

Reciprocal changes of expression between miR-17-5p and Stat3 in response to I/R-I or $\mathrm{H}_{2} \mathrm{O}_{2}$ damage

Significant upregulation of miR-17-5p expression was observed in mouse myocardium with I/R-I compared with the sham group $(P<0.05)$ (Fig. 1A). Intriguingly, Stat3 protein level was found consistently down-regulated in I/R-I (Fig. 1B), which was opposite to the changes of miR-17-5p.

Such changes in miR-17-5p and Stat 3 protein levels were reproduced in a cellular model of oxidative stress, established by treating NRVCs with $\mathrm{H}_{2} \mathrm{O}_{2}(100 \mu \mathrm{M})$ for $12 \mathrm{~h}$ (Fig. 1C \& 1D).

The inverse changes of miR-17-5p and Stat 3 protein levels suggested a possible targeting relationship between miR-17-5p and Stat3 and the involvement of these molecules in I/R-I 


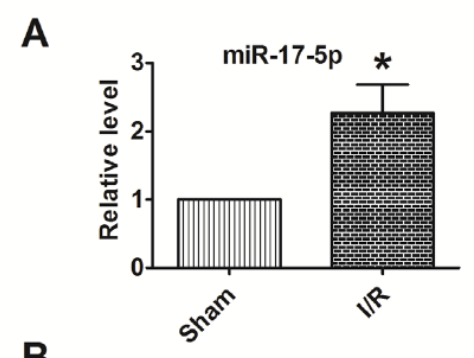

B

C

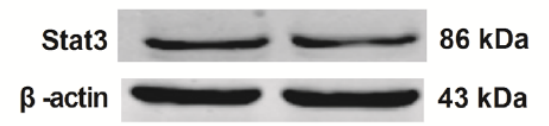

D
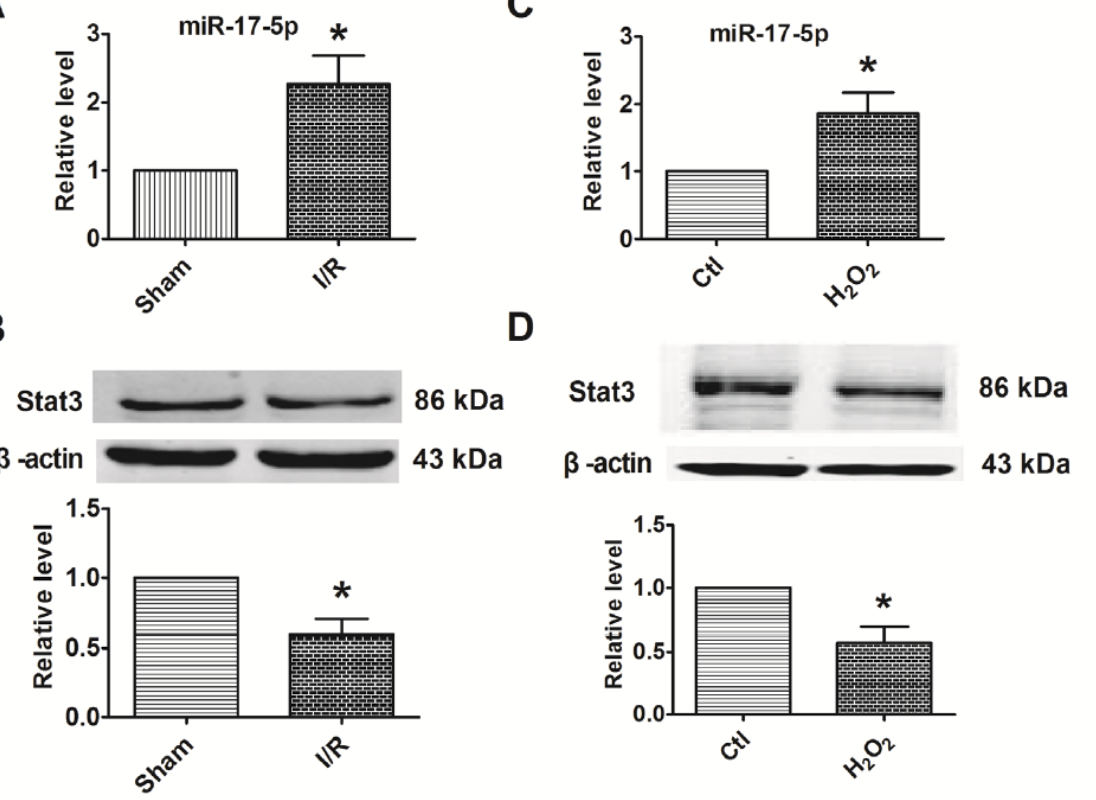

Fig. 1. Upregulation of miR-17-5p and downregulation of Stat3 protein in a mouse model of cardiac ischemia/reperfusion injury (I/R-I) and in a cellular model of cardiomyocyte injury induced by $\mathrm{H}_{2} \mathrm{O}_{2}$ in neonatal rat ventricular cells (NRVCs). (A) Real-time RT-PCR results showing the upregulation of miR-17-5p in I/R-I mice compared with sham groups. (B) Western blot analysis showing the downregulation of Stat3 protein level in I/R-I mice. (C) Real-time RT-PCR data showing the upregulation of miR-17-5p expression in NRVCs treated with $100 \mu \mathrm{M} \mathrm{H}_{2} \mathrm{O}_{2}$ for $12 \mathrm{~h}$. (D) Western blot analysis showing the downregulation of Stat3 protein level in NRVCs treated with $\mathrm{H}_{2} \mathrm{O}_{2} . \mathrm{n}=3$ mice or batches of cells; ${ }^{*} \mathrm{P}<0.05$ vs. Sham or Ctl.

associated with increased oxidative stress. These points were confirmed by the following experiments.

Overexpression of miR-17-5p aggravates cardiomyocyte apoptosis induced by $\mathrm{H}_{2} \mathrm{O}_{2}$

Our results with MTT assay showed that transfection of miR-17-5p alone into NRVCs did not affect cell viability under normal conditions (Fig. 2A). In line with these observations, TUNEL assay also demonstrated the lack of ability of miR-17-5p to induce apoptotic cell death (Fig. 2B). However, $\mathrm{H}_{2} \mathrm{O}_{2}(100 \mu \mathrm{M})$ reduced cell viability and miR-17-5p significantly exaggerated this detrimental change (Fig. 2C). In accordance with this effect, miR-17$5 \mathrm{p}$ also promoted oxidative stress-induced apoptosis of NRVCs, as reflected by enhanced DNA fragmentation determined by both TUNEL and ELISA assays (Fig. 2D \& 2E). Notably, inhibition of endogenous miR-17-5p by AM0-17-5p produced the opposite effect to miR17-5p: partially rescued the cell death induced by $\mathrm{H}_{2} \mathrm{O}_{2}$. And co-transfection of miR-17-5p with AM0-17-5p antagonized the apoptosis-promoting effect of miR-17-5p, indicating the specificity of miR-17-5p actions (Fig. 2D \& 2E). The cytoprotective effect against apoptotic cell death induced by $\mathrm{H}_{2} \mathrm{O}_{2}$ could be attributed to the knockdown of miR-17-5p expression (Fig. 2F).

\section{Inhibition of miR-17-5p attenuates $I / R-I$}

TUNEL staining confirmed the presence of apoptotic cells in a mouse model of I/R-I (Fig. $3 A$ ). If miR-17-5p plays a role in I/R-I, then inhibition of this miRNA should ameliorate it. To this end, we administered the LNA-antimiR-17-5p into mice through tail vein injection two 


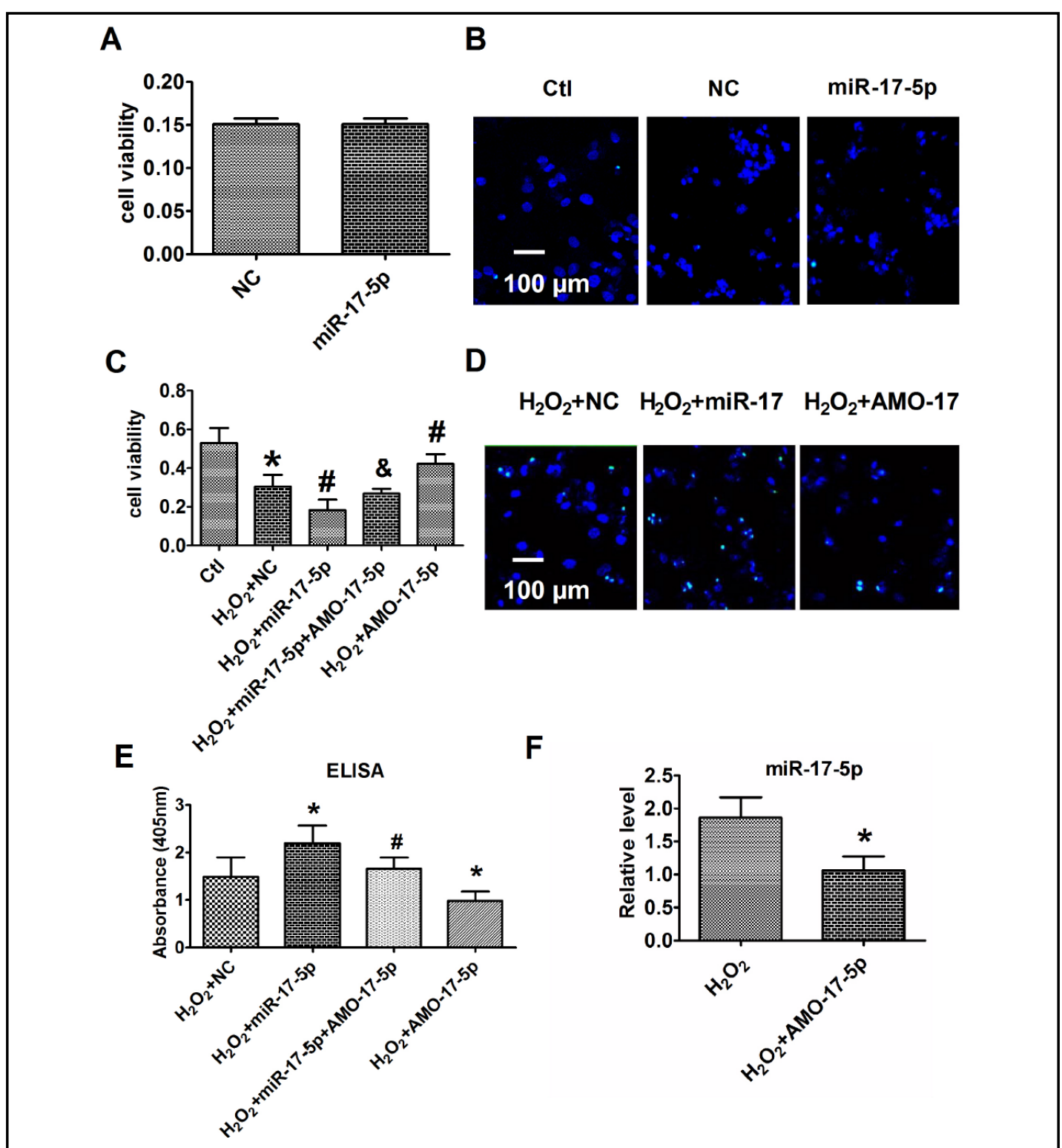

Fig. 2. miR-17-5p enhances cardiomyocyte apoptosis in response to $\mathrm{H}_{2} \mathrm{O}_{2}$. (A) NRVCs viability as detected by MTT assay showing the lack of ability of miR-17-5p alone to alter cell viability. (B) Representative images of TUNEL staining of NRVCs for DNA defragmentation showing the apoptotic cells (apoptotic cells stained in green and nucleus stained in blue with DAPI). (C) Detrimental effects of miR-17-5p on reduced cell viability induced by oxidative stress in NRVCs transfected with NC (negative control), miR-17-5p, AM0-17-5p, and miR-17-5p + AM0-17-5p for $24 \mathrm{~h}$, then treatment with $\mathrm{H}_{2} \mathrm{O}_{2}(100 \mu \mathrm{M})$ for $12 \mathrm{~h} . \mathrm{n}=8$ batches of cells; ${ }^{*} P<0.05$ vs. Ctl, ${ }^{\#} P<0.05$ vs. $\mathrm{H}_{2} \mathrm{O}_{2}+\mathrm{NC}$, ${ }^{\&} P<0.05$ vs. $\mathrm{H}_{2} \mathrm{O}_{2}+$ miR-17-5p. (D) Representative images of TUNEL staining of NRVCs showing the apoptotic cells. (E) Pro-apoptotic effect of miR-17-5p and anti-apoptotic effect of AM0-17-5p in response to $\mathrm{H}_{2} \mathrm{O}_{2}$, determined by ELISA quantification of DNA fragmentation expressed as absorbance values at $405 \mathrm{~nm} . \mathrm{n}=5$ batches of cells; ${ }^{*} P<0.05 v s . \mathrm{H}_{2} \mathrm{O}_{2}+\mathrm{NC}$; ${ }^{\#} P<0.05$ vs. $\mathrm{H}_{2} \mathrm{O}_{2}+$ miR-17-5p. (F) Verification of downregulation of miR-17-5p by AMO-17-5p in the presence of $\mathrm{H}_{2} \mathrm{O}_{2} . \mathrm{n}=5$ batches of cells, ${ }^{*} \mathrm{P}<0.05$ vs. $\mathrm{H}_{2} \mathrm{O}_{2} ; \mathrm{NC}$ indicates negative control.

days before I/R-I and measured apoptosis three days after LNA-antimiR-17-5p treatment. As shown in Fig. 3B, LNA-17-5p significantly reduced myocardial miR-17-5p level in the treated mice. More importantly, LNA-antimiR-17-5p treatment reduced the infarct size (Fig. 3C- 


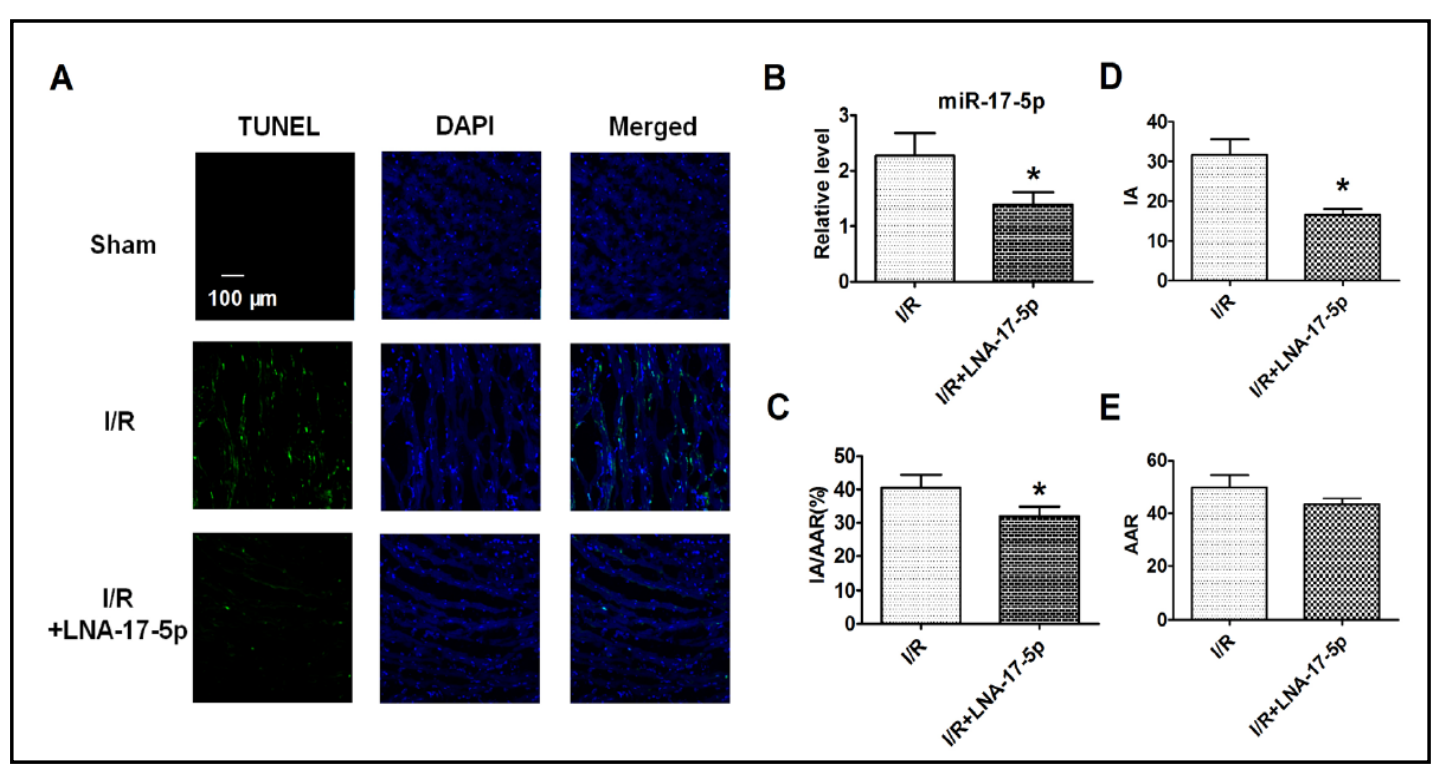

Fig. 3. Effects of LNA-17-5p on cardiomyocyte apoptosis in a mouse model of I/R-I. (A) TUNEL staining of apoptotic cells (apoptotic cells in green and nucleus in blue). (B) MiR-17-5p expression level in heart subjected to I/R after treatment with LNA-antimiR-17-5p, as determined by real-time RT-PCR. $n=6$ hearts in each group; ${ }^{*} P<0.05$ vs. I/R. (C) Statistical analysis of IA/AAR ratio for infarct areas detection. IA, infarct area; AAR, area at risk. $n=5$ hearts; ${ }^{*} P<0.05 v s$. I/R. Statistical analysis of IA (D) and AAR (E) for infarct areas detection. $\mathrm{n}=5$ hearts; ${ }^{*} P<0.05 \mathrm{vs}$. I/R.

3E). This beneficial effect of LNA-antimiR-17-5p could well be ascribed to its anti-apoptotic action via antagonizing miR-17-5p as the proportion of cells with positive TUNEL staining was substantially decreased by LNA-antimiR-17-5p in I/R-I mice (Fig. 3A).

Repression of Stat3 expression by miR-17-5p in a mouse model of $I / R-I$

As described in an earlier section, miR-17-5p and Stat3 demonstrated reciprocal changes in their levels in I/R-I mice and NRVCs under oxidative stress, indicating a targeting relationship between these two molecules. Indeed, by computational prediction using miRNA databases, we identified two miR-17-5p seed motif-matching sites with favorable energy profiles in the 3'UTR of Stat3 (Fig. 4A). Stat3, a cellular survival factor, is increased in response to oxidative stress and plays an important antiapoptotic role in cardiomyocytes [28]. In our previous study, we identified a population of cells bearing higher resistance to apoptosis and found that the cells that survived from apoptotic insults had markedly higher levels of Stat3 [29]. To experimentally establish Stat3 as a target gene for miR-17-5p, we performed luciferase reporter activity assay in HEK293 cells with the vectors engineered to carry the 3'UTR of Stat3. Transfection of miR-17-5p reduced luciferase reporter activity (Fig. 4B). Co-transfection with the AM0-17-5p reversed the inhibitory effects and application of the AMO-17-5p alone to knockdown endogenous miR-17-5p increased luciferase activity (Fig. 4B). The targeting was confirmed at the protein level by Western blot analysis in NRVCs, in particular, transfection of miR-17-5p markedly decreased Stat3 protein level and this effect was abolished by AMO-17-5p. In addition, transfection of AMO-17-5p alone significantly increased Stat3 protein expression (Fig. 4C). These results demonstrated Stat3 was a direct target of miR-17-5p and the detrimental action of miR-17-5p in I/R-I and $\mathrm{H}_{2} \mathrm{O}_{2}$ treated NRVCs might well be conferred by this targeting relationship.

Stat3 is a transcription factor that is activated by a wide variety of external stimuli, including cytokines, hormones, growth factors, and translocated to the nucleus leading to activation of gene transcription, such as Bcl-2 and Mcl-1 [21]. Activation of Stat3 by phosphorylation (p-Stat3) attenuates I/R-induced mitochondrial oxidative damage after 
A

$$
\text { Site 1: }
$$$$
\begin{aligned}
& \text { hsa-miR-17-5p } \\
& \text { 3'-GAUGGACGUGACAUUCGUGAAAC -5' }
\end{aligned}
$$$$
\begin{aligned}
& \text { 5'-CUUUGAGCAAUCUGGGCACUUUU-3' } \\
& \text { II I I I I I I III }
\end{aligned}
$$$$
\text { 158-164 of STAT3 3' UTR }
$$$$
\text { Site 2: }
$$$$
\text { hsa-miR-17-5p }
$$$$
\text { 3'-GAUGGACGUGACAUUCGUGAAAC -5' }
$$

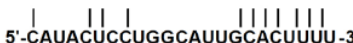$$
\text { 448-454 of STAT3 3' UTR }
$$

Site 1:

3'-GAUGGACGMUR RACACAUUCGUGAAAC -5'

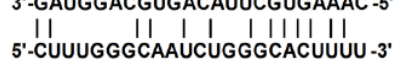
157-163 of STAT3 3' UTR

Site 2: mmu-miR-17-5p

3'-GAUGGACGUGACAUUCGUGAAAC -5'

5'-GGAACUCCUGGCUCUGCACUUUC -3'

B

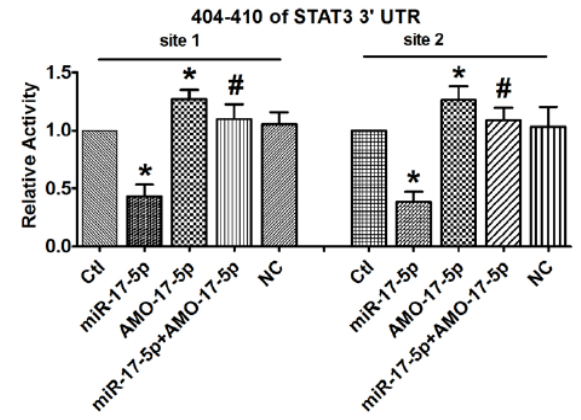

C

D
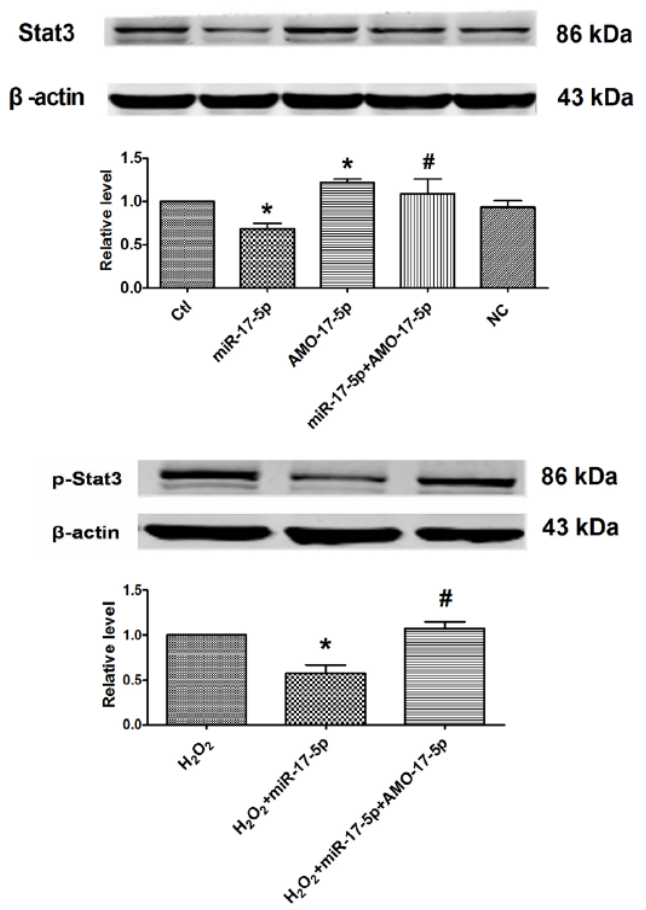

Fig. 4. Repression of Stat3 expression by miR-17-5p. (A) Sequence alignment showing complementarity between miR-17-5p and the two putative binding sites in the 3'UTR of the Stat3 gene. (B) The interactions between miR-17-5p and its binding sites in the 3'UTR of Stat3 were measured by luciferase reporter gene assay in HEK293 cells. Site 1 and site 2 refer to the first and second binding sites for miR-17-5p in the 3'UTR of the Stat3 gene, respectively. (C) Western blot analysis showing the effects of miR-17-5p on Stat3 protein level. (D) The expression of $p$-Stat 3 was determined by Western blot analysis. $n=4$ batches of cells; ${ }^{*} P<0.05$ vs. Ctl or $\mathrm{H}_{2} \mathrm{O}_{2}$; ${ }^{\#} P<0.05$ vs. miR-17-5p or $\mathrm{H}_{2} \mathrm{O}_{2}+$ miR-17-5p.

melatonin treatment through up-regulating the anti-apoptotic protein Bcl-2 [22]. Inhibition of p-Stat3 is thus expected to result in a loss of the intrinsic anti-apoptotic property of cells. Our data shown in Fig. 4D demonstrated overexpression of miR-17-5p significantly decreased p-Stat 3 expression in response to oxidative stress in NRVCs, and this effect was abrogated by co-transfection of AMO-17-5p.

\section{Discussion}

Cardiomyocyte apoptosis contributes critically to cardiac I/R-I [30]. It has been well established that multiple signaling pathways are activated during I/R-I, such as oxidative stress, calcium overload and ATP depletion, resulting in a net outcome of increased apoptotic cell death [31, 32]. Here we showed that miR-17-5p promoted cardiomyocyte apoptosis induced by oxidative stress via targeting Stat3 in both animal model of I/R-I and cellular model of cardiomyocyte injury induced by oxidative stress.

MiR-17-5p is a member of the miR-17-92 cluster, located in the third intron of a $\sim 7 \mathrm{~kb}$ primary transcript known as C13orf25 or MIR17HG1 and has been shown to regulate tumor proliferation and progression $[13,14]$. Data from numerous studies point to the suppression of apoptosis of cancer cells as the primary mechanism for the oncogenic action of miR-17- 
$5 p$. The oncogenic activity of the miR-17-92 cluster is ascribed to downregulation of tumor suppressors retinoblastoma 1 and the cell cycle inhibitor P21, as well as the pro-apoptotic inhibitor Bim [33]. On the other hand, a few studies suggested the opposite: miR-17-5p could also act as a tumor suppressor through targeting E2F, a potent oncogene promoting cell cycle progression by targeting downstream regulators [34-35]. Notably miR-17-5p was found activated during the recovery phase of kidney I/R in mice [20].

In this study, we demonstrated that miR-17-5p was upregulated under I/R-I and oxidative stress and this upregulation promoted I/R-I or oxidative stress-induced apoptotic cell death. Furthermore, knockdown of endogenous miR-17-5p by LNA-miR-17-5p or AMO17-5p consistently reduced the cardiomyocyte apoptosis and death in response to I/R. The mechanism for the pro-apoptotic property of miR-17-5p is likely due to the gene targeting of the cell survival mediator Stat3.

It is known that activation of Stat3 by phosphorylation increases cell survival and resistance to apoptosis in response to various cytokines and stress stimuli. The cytoprotective effect is ascribed to the pro-transcription activity of Stat3 for downstream molecules, including Bcl-2 family proteins and Mcl-1 which in turn produce anti-apoptotic effects [36]. Dziennis et al. observed accumulation of p-Stat 3 in the peri-infarct region after treating with estradiol inhibited cerebral ischemic damage in oophorectomy rats [37]. We have previously demonstrated that transactivation of Jak2/Stat 3 under oxidative stress confers cells higher resistance to apoptosis, whereas knockdown of Stat3 by siRNA or inhibition of Stat3 activity promotes $\mathrm{H}_{2} \mathrm{O}_{2}$-induced cardiomyocyte injury [29]. In the present study, we have experimentally established Stat3 as a direct target gene for miR-17-5p. Overexpression of miR-17-5p significantly reduced p-Stat3 level in both in vivo setting with a mouse model of I/R-I and in vitro setting with a cellular model of oxidative stress. Co-application of AMO17-5p prevented this downregulation.

Apoptosis is an essential process for developmental morphogenesis by regulating cell homeostasis and tissue remodeling in organisms and thus can be considered as a physiological regulator of cell density. Nonetheless excessive apoptotic cell death is deemed to cause pathogenesis of degenerative diseases like myocardial infarction, failing heart, atherosclerotic arteries and hypertensive vessels, and Alzheimer's disease and other neurodegenerative disorders [38]. The heart is composed of post-mitotic cells that cannot divide and new cardiomyocytes cannot be regenerated. Under such a circumstance, preserving cells that can survive from apoptotic injuries become the only hope for delaying pathological processes associated with cell death. Indeed, cells of even the same histological origins possess different susceptibility or resistance to apoptotic insults; some can tolerate more severe apoptotic induction and some cannot. This property is important for normal morphogenesis during development and for abnormal loss of cells during pathogenesis. Obviously, understanding signal transduction processes in cells that survived from apoptotic injuries is of crucial importance to develop better strategies of preserving cells with apoptosis resistance.

Collectively, our findings revealed that miR-17-5p mediated the apoptotic signal of oxidative stress and contributed to the I/R-I and possibly to other pathological conditions as well associated with oxidative stress such as heart failure. Furthermore, our results suggest that precaution should be taken when using this miRNA as a target for anti-cancer therapy for they may cause cardiac complications.

\section{Acknowledgement}

This work was supported in part by the Major Program of National Natural Science Foundation of China (81130088, 81230081), 973 Program Earlier Research Project (2012CB723505), and the National Nature Science Foundation of China (81170219 and 81370245). 
Du et al.: miRNA-17-5p Promotes Cardiomyocyte Apoptosis

\section{Disclosure Statement}

The authors declare that they have no conflict of interests.

\section{References}

$\rightarrow 1$ Zornoff LA, Paiva SA, Duarte DR, Spadaro J: Ventricular remodeling after myocardial infarction: Concepts and clinical implications. Arq Bras Cardiol 2009;92:150-164.

2 Katircioglu SF, Iscan HZ, Ulus T, Saritas Z: Myocardial preservation in acute coronary artery occlusion with coronary sinus retroperfusion and carnitine. J Cardiovasc Surg (Torino) 2000;41:45-50.

- Z Zweier JL: Measurement of superoxide-derived free radicals in the reperfused heart. Evidence for a free radical mechanism of reperfusion injury. J Biol Chem 1988;263:1353- 1357.

4 Garlick PB, Davies MJ, Hearse DJ, Slater TF: Direct detection of free radicals in the reperfused rat heart using electron spin resonance spectroscopy. Circ Res 1987;61:757-760.

5 Arroyo CM, Kramer JH, Dickens BF, Weglicki WB: Identification of free radicals in myocardial ischemia/ reperfusion by spin trapping with nitrone dmpo. FEBS Letters 1987;221:101-104.

6 McCully JD, Wakiyama H, Hsieh YJ, Jones M, Levitsky S: Differential contribution of necrosis and apoptosis in myocardial ischemia-reperfusion injury. Am J Physiol Heart Circ Physiol 2004;286:H1923-1935.

7 Abe T, Zhou P, Jackman K, Capone C, Casolla B, Hochrainer K, Kahles T, Ross ME, Anrather J, Iadecola C: Lipoprotein receptor-related protein-6 protects the brain from ischemic injury. Stroke 2013;44:2284-2291.

8 Krol J, Loedige I, Filipowicz W: The widespread regulation of microrna biogenesis, function and decay. Nat Rev Genet 2010;11:597-610.

-9 Norbury CJ: 3' Uridylation and the regulation of RNA function in the cytoplasm. Biochem Soc Trans. 2010;38:1150-1153.

10 Ren XP, Wu J, Wang X, Sartor MA, Qian J, Jones K, Nicolaou P, Pritchard TJ, Fan GC: MicroRNA-320 is involved in the regulation of cardiac ischemia/reperfusion injury by targeting heat-shock protein 20. Circulation 2009;119:2357-2366.

11 Wang X, Zhang X, Ren XP, Chen J, Liu H, Yang J, Medvedovic M, Hu Z, Fan GC: MicroRNA-494 targeting both proapoptotic and antiapoptotic proteins protects against ischemia/reperfusion-induced cardiac injury. Circulation 2010;122:1308-1318.

12 Pan Z, Sun X, Ren J, Li X, Gao X, Lu C, Zhang Y, Sun H, Wang Y, Wang H, Wang J, Xie L, Lu Y, Yang B: MiR-1 exacerbates cardiac ischemia-reperfusion injury in mouse models. PLoS One 2012;7:e50515.

13 Mogilyansky E, Rigoutsos I: The miR-17/92 cluster: A comprehensive update on its genomics, genetics, functions and increasingly important and numerous roles in health and disease. Cell Death Differ 2013;20:1603-1614.

-14 Zhou L, Qi RQ Liu M, Xu YP, Li G, Weiland M, Kaplan DH, Mi QS: MicroRNA miR-17-92 cluster is highly expressed in epidermal langerhans cells but not required for its development. Genes Immun 2014;15:5761.

15 Chen J, Huang ZP, Seok HY, Ding J, Kataoka M, Zhang Z, Hu X, Wang G, Lin Z, Wang S, Pu WT, Liao R, Wang DZ: MiR-17-92 cluster is required for and sufficient to induce cardiomyocyte proliferation in postnatal and adult hearts. Circ Res 2013;112:1557-1566.

-16 Yu J, Ohuchida K, Mizumoto K, Fujita H, Nakata K, Tanaka M: MicroRNA miR-17-5p is overexpressed in pancreatic cancer, associated with a poor prognosis, and involved in cancer cell proliferation and invasion. Cancer Biol Ther 2010;10:748-757.

17 Takakura S, Mitsutake N, Nakashima M, Namba H, Saenko VA, Rogounovitch TI, Nakazawa Y, Hayashi T, Ohtsuru A, Yamashita S: Oncogenic role of miR-17-92 cluster in anaplastic thyroid cancer cells. Cancer Sci 2008;99:1147-1154.

18 Zhang B, Pan X, Cobb GP, Anderson TA: MicroRNAs as oncogenes and tumor suppressors. Dev Biol 2007;302:1-12.

19 Danielson LS, Park DS, Rotllan N, Chamorro-Jorganes A, Guijarro MV, Fernandez-Hernando C, Fishman GI, Phoon CK, Hernando E: Cardiovascular dysregulation of miR-17-92 causes a lethal hypertrophic cardiomyopathy and arrhythmogenesis. FASEB J 2013;27:1460-1467. 


\section{Cellular Physiology Cell Physiol Biochem 2014;34:955-965

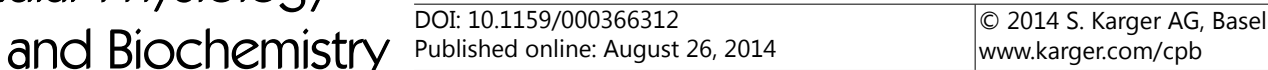 \\ Du et al.: miRNA-17-5p Promotes Cardiomyocyte Apoptosis}

20 Kaucsar T, Revesz C, Godo M, Krenacs T, Albert M, Szalay CI, Rosivall L, Benyo Z, Batkai S, Thum T, Szenasi G, Hamar P: Activation of the miR-17 family and miR-21 during murine kidney ischemia-reperfusion injury. Nucleic Acid Ther 2013;23:344-354.

21 Yeh CT, Huang WC, Rao YK, Ye M, Lee WH, Wang LS, Tzeng DT, Wu CH, Shieh YS, Huang CY, Chen YJ, Hsiao M, Wu AT, Yang Z, Tzeng YM: A sesquiterpene lactone antrocin from antrodia camphorata negatively modulates Jak2/Stat3 signaling via microRNA let-7c and induces apoptosis in lung cancer cells. Carcinogenesis 2013;34:2918-2928.

22 Yang Y, Duan W, Jin Z, Yi W, Yan J, Zhang S, Wang N, Liang Z, Li Y, Chen W, Yi D, Yu S: Jak2/Stat3 activation by melatonin attenuates the mitochondrial oxidative damage induced by myocardial ischemia/reperfusion injury. J Pineal Res 2013;55:275-286.

-23 Vandeput F, Krall J, Ockaili R, Salloum FN, Florio V, Corbin JD, Francis SH, Kukreja RC, Movsesian MA: Cgmphydrolytic activity and its inhibition by sildenafil in normal and failing human and mouse myocardium. J Pharmacol Exp Ther 2009;330:884-891.

-24 Li YS, Gao BR: Transplantation of neonatal cardiomyocytes plus fibrin sealant restores myocardial function in a rat model of myocardial infarction. Chin Med J (Engl) 2007;120:2022-2027.

25 Yang B, Lin H, Xiao J, Lu Y, Luo X, Li B, Zhang Y, Xu C, Bai Y, Wang H, Chen G, Wang Z: The muscle-specific microRNA miR-1 regulates cardiac arrhythmogenic potential by targeting GJA1 and KCNJ2. Nat Med 2007;13:486-491.

-26 Jiang J, Lee EJ, Gusev Y, Schmittgen TD: Real-time expression profiling of microRNA precursors in human cancer cell lines. Nucleic Acids Res 2005;33:5394-5403.

27 Cebotari S, Mertsching H, Kallenbach K, Kostin S, Repin O, Batrinac A, Kleczka C, Ciubotaru A, Haverich A: Construction of autologous human heart valves based on an acellular allograft matrix. Circulation 2002;106:I63-I68.

-28 Ng IH, Yeap YY, Ong LS, Jans DA, Bogoyevitch MA: Oxidative stress impairs multiple regulatory events to drive persistent cytokine-stimulated Stat3 phosphorylation. Biochim Biophys Acta 2014;1843:483-94.

29 Lu Y, Zhou J, Xu C, Lin H, Xiao J, Wang Z, Yang B: Jak/Stat and PI3K/Akt pathways form a mutual transactivation loop and afford resistance to oxidative stress-induced apoptosis in cardiomyocytes. Cell Physiol Biochem 2008;21:305-314.

30 Chien CT, Chen CF, Hsu SM, Lee PH, Lai MK: Protective mechanism of preconditioning hypoxia attenuates apoptosis formation during renal ischemia/reperfusion phase. Transplant Proc 1999;31:2012-2013.

-31 Vanlangenakker N, Vanden Berghe T, Krysko DV, Festjens N, Vandenabeele P: Molecular mechanisms and pathophysiology of necrotic cell death. Curr Mol Med 2008;8:207-220.

-32 Halestrap AP: Calcium, mitochondria and reperfusion injury: A pore way to die. Biochem Soc Trans 2006;34:232-237.

33 Spaccarotella E, Pellegrino E, Ferracin M, Ferreri C, Cuccuru G, Liu C, Iqbal J, Cantarella D, Taulli R, Provero P, Di Cunto F, Medico E, Negrini M, Chan WC, Inghirami G, Piva R: Stat3-mediated activation of microRNA cluster 17 92 promotes proliferation and survival of ALK-positive anaplastic large cell lymphoma.

Haematologica 2014;99:116-24.

-34 Novotny GW, Sonne SB, Nielsen JE, Jonstrup SP, Hansen MA, Skakkebaek NE, Rajpert-De Meyts E, Kjems J, Leffers H: Translational repression of E2F1 mRNA in carcinoma in situ and normal testis correlates with expression of the miR-17-92 cluster. Cell Death Differ 2007;14:879-882.

35 Cloonan N, Brown MK, Steptoe AL, Wani S, Chan WL, Forrest AR, Kolle G, Gabrielli B, Grimmond SM: The miR-17-5p microRNA is a key regulator of the G1/S phase cell cycle transition. Genome Biol 2008;9:R127.

-36 Bhattacharya S, Ray RM, Johnson LR: Stat3-mediated transcription of Bcl-2, Mcl-1 and c-IAP2 prevents apoptosis in polyamine-depleted cells. Biochem J 2005;392:335-344.

37 Dziennis S, Jia T, Ronnekleiv OK, Hurn PD, Alkayed NJ: Role of signal transducer and activator of transcription-3 in estradiol-mediated neuroprotection. J Neurosci 2007;27:7268-7274.

38 Arribas SM, Hermida C, Gonzalez MC, Wang Y, Hinek A: Enhanced survival of vascular smooth muscle cells accounts for heightened elastin deposition in arteries of neonatal spontaneously hypertensive rats. Exp Physiol 2010;95:550-560. 